\title{
Development of Drug-Loaded PLGA Microparticles with Different Release Patterns for Prolonged Drug Delivery
}

\author{
Yeonsoon Choi, Jae-ryang Joo, Areum Hong, and Jong-Sang Park* \\ Department of Chemistry, College of Natural Science, Seoul National University, Seoul 151-742, Korea \\ *E-mail: pfjspark@plaza.snu.ac.kr \\ Received November 29, 2010, Accepted January 4, 2011
}

\begin{abstract}
For the prolonged delivery and sustained release rates of low molecular weight drugs, poly(lactic-co-glycolic acid) (PLGA) microparticles containing the drug SKL-2020 have been investigated. On increasing polyvinyl alcohol (PVA) concentration (from $0.2 \%$ to $5 \%$ ), the size of microparticles decreased (from $48.02 \mu \mathrm{m}$ to 10.63 $\mu \mathrm{m})$ and more uniform size distribution was noticeable due to the powerful emulsifying ability of PVA. A higher drug loading (from 5\% to 20\%) caused a larger concentration gradient between 2 phases at the polymer precipitation step; this resulted in decreased encapsulation efficiency (from 34.19\% to 25.67\%) and a greater initial burst (from 61.71\% to 70.05\%). SKL-2020-loaded PLGA microparticles prepared with different fabrication conditions exhibited unique release patterns of SKL-2020. High PVA concentration and high drug loading led to an initial burst effect by rapid drug diffusion through the polymer matrix. Since PLGA microparticles enabled the slow release of SKL-2020 over 1 week in vitro and in vivo, more convenient and comfortable treatment could be facilitated with less frequent administration. It is feasible to design a release profile by mixing microparticles that were prepared with different fabrication conditions. By this method, the initial burst could be repressed properly and drug release rate could decrease.
\end{abstract}

Key Words : Drug delivery system, Biodegradable polymers, PLGA microparticles, Solvent evaporation method

\section{Introduction}

Polymeric microparticles have been employed for decades in drug delivery to specific sites while maintaining desirable release patterns. ${ }^{1}$ Biodegradable polymers such as poly(lactic-co-glycolic acid) (PLGA) or poly(lactic acid) (PLA) have been considered for this purpose, because of their biocompatibility and proven safety. PLGA is a Food and Drug Administration (FDA)-approved biodegradable polymer. PLGA ester linkages degrade by hydrolysis in the presence of water, rendering it biocompatible. Because the time required for degradation of PLGA is different depending on the ratio of monomers (lactide and glycolide), the biodegradability may be programmable., ${ }^{2,3}$ A higher glycolide unit content results in decreased degradation time.

To design a drug delivery system, fabrication methods should be optimized because microparticle characteristics are affected by fabrication methods. Various methods of drug-encapsulated microparticles fabrication have been studied intensively. ${ }^{3-6}$ An oil-in-water (o/w) solvent extraction/evaporation technique is one of the most widely-used methods for particle formation and drug encapsulation due to the convenience of the process. ${ }^{7,8}$ This method consists of 3 major steps: dissolving the drug and polymer into an organic solvent, dispersing them into water and evaporating or extracting the solvent until particles are formed. First, an oil phase is dispersed as droplets by rapid stirring, followed by drug encapsulation by the precipitated polymer.

SKL-2020 is a newly synthesized drug developed by the SK Corporation (Daejeon, Korea) and is known to play a role in endothelial cell proliferation and differentiation. The biggest challenge in the use of this drug is its short half-life of less than $30 \mathrm{~min}$ in animal bodies; additionally, drug concentration in the blood tends to decrease significantly. For prolonged drug delivery in vivo, the drug delivery system is critical; for example, polymeric microparticles can provide sustained drug release at a slow rate. A long-term delivery system can minimize side effects caused by frequent drug administration and provide patients better comfort and convenience. For an efficient and optimized drug delivery system, many key factors must be considered such as morphology, particle size, encapsulation efficiency and most importantly, release properties. It is typically advantageous to obtain a desirable release rate and period through controlled particle size.

In this study, various SKL-2020-encapsulated PLGA microparticles were fabricated and studied to determine the optimal release profile in vivo. The effects of initial drug loading amount and polyvinyl alcohol (PVA) concentration on the release profiles were investigated to determine encapsulation efficiency and initial burst effect. To design appropriate release patterns of microparticles, we also used mixtures of microparticles prepared with different fabrication conditions.

\section{Materials and Methods}

Materials. SKL-2020 was supplied from the SK Corporation (Daejeon, Korea). PLGA (RG504H, 50:50, molecular weight (MW) 40,000-70,000) was obtained from Boehringer 
Ingelheim (Ingelheim, Germany) and PVA (MW 30,00070,000) was obtained from Sigma (St. Louis, MO, USA). All other reagents were analytical and high-performance liquid chromatography (HPLC) grade and used without further purification.

\section{Methods.}

Preparation of SKL-2020-Loaded PLGA Microparticles. PLGA microparticles containing SKL-2020 were prepared by an oil-in-water $(\mathrm{o} / \mathrm{w})$ emulsion solvent evaporation method. Solution containing $500 \mathrm{mg}$ of PLGA and varying amounts of SKL-2020 (100 mg, $50 \mathrm{mg}$, and $25 \mathrm{mg}$ ) were prepared by dissolving them in $10 \mathrm{ml}$ of methylene chloride. The solution was emulsified in $100 \mathrm{ml}$ of PVA aqueous solutions of varying concentration $(0.2 \%, 2 \%$, and $5 \% \mathrm{w} / \mathrm{v}$, respectively) by stirring at $800 \mathrm{rpm}$ to evaporate the organic solvent and form SKL-2020-loaded PLGA microparticles. After $3 \mathrm{~h}$ of stirring, the aqueous suspension was centrifuged and washed with distilled water twice and freeze-dried.

Morphological Characterization. The surface morphology and size of SKL-2020-loaded PLGA microparticles were examined using scanning electron microscopy (SEM, JSM-840A, JEOL, Tokyo, Japan). The samples were placed on an aluminum stub and coated with platinum for $300 \mathrm{~s}$ before taking photographs at an accelerating voltage of 15 $\mathrm{kV}$.

Particle Size Analysis. The size of SKL-2020-loaded PLGA microparticles was estimated using SEM images. The pictures were analyzed using ImageJ $1.33 \mathrm{u}$ (available at http://rsb.info.nih.gov/ij/) software to calculate individual microparticle diameters and mean values.

Evaluation of Drug Content and Encapsulation Efficiency. The drug content and encapsulation efficiency of SKL-2020-loaded PLGA microparticles was measured by UV spectrometry at $287 \mathrm{~nm}$ (UV-1601PC, Shimadzu, Kyoto, Japan). SKL-2020-loaded PLGA microparticles $(n=3)$ were dissolved in $5 \mathrm{~mL}$ of DMSO. The contents of SKL-2020 were determined by using UV absorbance of microparticles in DMSO. The encapsulation efficiency of PLGA microparticles was calculated as the ratio of actual-to-theoretical drug content.

In vitro Drug Release Studies. In vitro release profiles of PLGA microparticles containing SKL-2020 were obtained using a UV spectrometer at $287 \mathrm{~nm}$ (UV-1601PC, Shimadzu, Kyoto, Japan). A solution of $0.05 \%$ (w/v) Tween20/PBS pH 7.4 was used as the release media. SKL-2020-loaded PLGA microparticles $(n=3)$ were suspended in $10 \mathrm{~mL}$ of the release media and incubated at $37{ }^{\circ} \mathrm{C}$ with continuous vertical rotation. After centrifugation, $0.5 \mathrm{~mL}$ of supernatant was extracted from sample tubes at specified time intervals ( $2 \mathrm{~h}$, and $8 \mathrm{~h}$, and $1,2,4,7$, and 9 days). The amount of SKL-2020 in each sample was divided by the total amount of SKL-2020 in microparticles to give a cumulative percentage. The experiments were repeated 3 times.

In vivo Drug Release Studies. This study was reviewed and approved by the Institutional Animal Care and Use Committee (IACUC) of Samsung Biomedical Research Institute (SBRI). SBRI is an Association for Assessment and
Accreditation of Laboratory Animal Care International (AAALAC International) accredited facility and abides by the Institute of Laboratory Animal Resources (ILAR) guide. $\mathrm{Balb} / \mathrm{C}$ mice aged 8 weeks were anesthetized with sodium ketamine and xylene. SKL-2020-loaded PLGA microparticles were dissolved in phosphate-buffered saline (PBS, $\mathrm{pH}$ 7.4). This solution was injected into two spots at adductor of mice. After injection, the adductor muscles were excised at specified time intervals $(0,2,4$, and 7 days). Hind limb samples were snap-frozen in liquid nitrogen immediately after harvest and stored at $-80{ }^{\circ} \mathrm{C}$ until extraction.

\section{Results and Discussion}

Morphology of SKL-2020-Loaded PLGA Microparticles. The morphology of SKL-2020-loaded PLGA microparticles with different PVA concentrations was observed by SEM (Fig. 1). Microparticle morphology is typically affected by the rate of polymer precipitation at the solvent removal step. ${ }^{9}$ When the organic solvent is removed slowly, the polymer can precipitate gradually and generate a smooth surface. However, rapid evaporation of organic solvent at a high temperature typically leads to a porous surface.

(a)

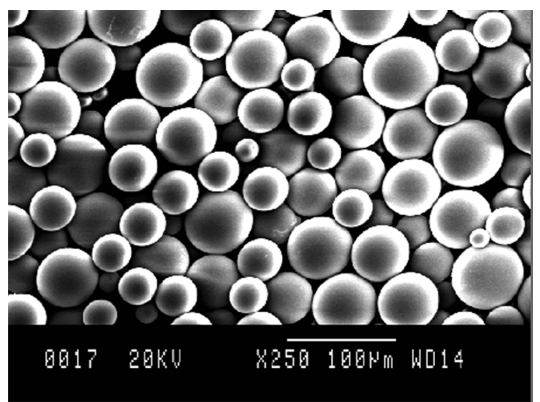

(b)

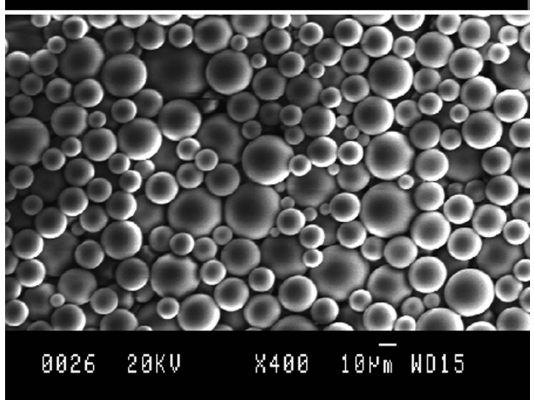

(c)

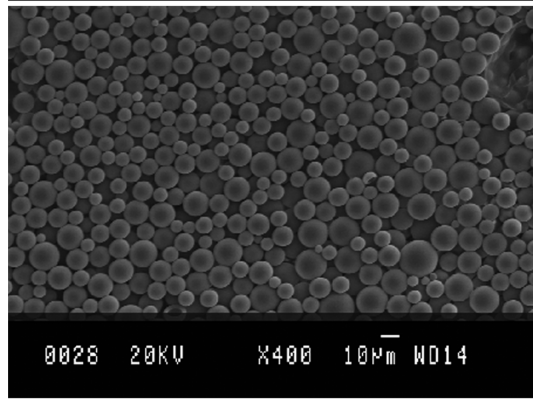

Figure 1. Scanning electron microscopy morphology of SKL2020-loaded PLGA microparticles fabricated with different PVA concentrations. (a) $0.2 \%$ PVA solution (b) 2\% PVA solution (c) 5\% PVA solution 
According to SEM data, SKL-2020-loaded PLGA microparticles had a perfect spherical shape with a smooth surface and no pores or dented portions, regardless of size and preparation conditions such as PVA concentration. In addition to polymer precipitation rate, PVA presence may contribute to this smooth surface because of its powerful emulsion-stabilizing effect.

PVA Concentration. The role of PVA as a surfactant and a stabilizer in manufacturing polymeric particles has been widely studied. After the oil phase is dispersed into the water phase, PVA stabilizes small emulsion droplets to prevent coalescence. With high PVA concentration, particle size generally decreases as shear stress and viscosity increase. Since PVA concentration is a key factor in controlling particle size and release kinetics, variation of PVA concentration provides an opportunity to study the relationship between particle size and release profile. Thus, we fabricated 3 groups of SKL-2020-loaded PLGA microparticles prepared in $0.2 \%, 2 \%$, and $5 \%(\mathrm{w} / \mathrm{v})$ PVA solution. Other preparation conditions, such as initial drug ratio $(5 \%)$ and stirring rate $(800 \mathrm{rpm})$, were the same for all 3 groups. The mean size of fabricated microparticles decreased (48.02, 16.95 , and $10.63 \mu \mathrm{m}$ ) with increasing PVA concentration (Table 1). The size distributions of the 3 groups are also illustrated in Figure 2. It should be noted that PVA concentration also affected the size distribution of microparticles. While SKL-2020-loaded PLGA microparticles prepared in $0.2 \%(\mathrm{w} / \mathrm{v})$ PVA solution showed broad size distribution from $15-20 \mu \mathrm{m}$ to $70-75 \mu \mathrm{m}$, microparticles prepared in $2 \%$ and 5\% (w/v) PVA solution displayed a much narrower size distribution. This tendency was confirmed by SEM data. In Figures $1(a)$ and (b), the primary difference in microparticle size is observed. In Figure 1(c), relatively uniform microparticle generation can be seen.

In the designing of a drug release system, the amount of drug release is inversely proportional to particle size. Small particles generally release encapsulated drugs quickly and result in a very large initial burst due to the short diffusion path length and large surface area. However, it is not always accurate to generalize in vitro release patterns by varying PVA concentration. Depending on manufacturing methods, encapsulation efficiency or burst effect can be different even with the same PVA concentration, resulting in different release properties. Yang et al. reported that larger microparticles fabricated by the double-emulsion solvent extraction/evaporation method with a low PVA concentration in the external water phase have a more rapid bovine serum albumin (BSA) release. ${ }^{10} \mathrm{PVA}$ concentration may affect many

Table 1. Characterization of SKL-2020-loaded PLGA microparticles fabricated with different PVA concentrations

\begin{tabular}{ccccc}
\hline $\begin{array}{c}\text { PVA conc. } \\
(\mathrm{w} / \mathrm{v})\end{array}$ & $\begin{array}{c}\text { Final drug } \\
\text { content }(\%)\end{array}$ & E.E. $(\%)$ & $\begin{array}{c}\text { Mean size } \\
(\mu \mathrm{m})\end{array}$ & $\begin{array}{c}\text { Burst } \\
(\%)\end{array}$ \\
\hline $0.2 \%$ & 2.15 & 45.17 & 48.02 & 19.68 \\
$2 \%$ & 1.83 & 38.49 & 16.95 & 29.57 \\
$5 \%$ & 1.63 & 34.19 & 10.63 & 58.62 \\
\hline
\end{tabular}

parameters, such as viscosity of the water phase, stability of emulsion droplets, or drug distribution within microparticles besides particle size.

Released SKL-2020 from the 3 groups of microparticles was detected by UV spectrometry at specified time intervals. Additionally, encapsulation efficiency was calculated (Table 1). Given that the initial drug content was $5 \%$ for all 3
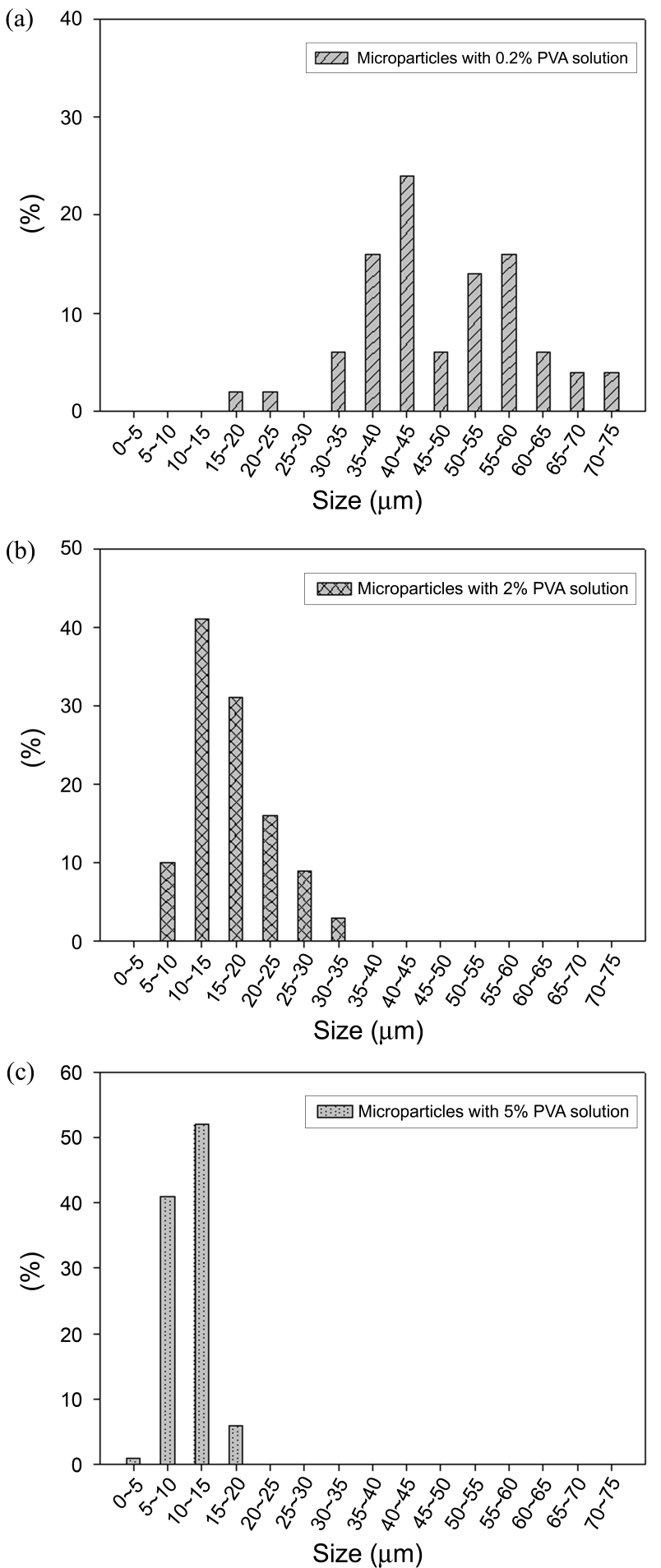

Figure 2. Size distribution of SKL-2020-loaded PLGA microparticles fabricated with different PVA concentrations. (a) $0.2 \%$ PVA solution (b) 2\% PVA solution (c) 5\% PVA solution 
groups, final drug content solely depends on encapsulation efficiency. As PVA concentration increases, encapsulation efficiency decreases as does the final drug content. According to Yeo et al. in 2004, there are numerous parameters relevant to encapsulation efficiency such as polymer solubility in organic solvent, organic solvent solubility in water, polymer concentration, solvent removal rate, and interaction between drug and polymer, among others. ${ }^{9}$ Because SKL2020 is relatively hydrophilic and the solvent removal rate by stirring is low, encapsulation efficiency of SKL-2020loaded PLGA microparticles is typically less than $50 \%$. This means more than $50 \%$ of the initially loaded drug is lost to the water phase by diffusion, before polymer precipitation. The decreasing tendency of encapsulation efficiency with increasing PVA concentration may be based on increasing surface area of PLGA microparticles. Since drug loss occurs when oil phase remains in a semi-solid state before polymer hardening, the drug can diffuse out quickly when the surface area of microparticles is large. Although emulsion droplets in a water phase are significantly more stable in high PVA concentration, the size and surface area of particles in the transitional state determine the amount of encapsulated drug.

Based on encapsulation efficiency, release patterns of 3 microparticle groups were obtained (Fig. 3). By exhibiting a large difference in initial burst, the 3 groups displayed unique release patterns of SKL-2020-loaded PLGA microparticles. Initial burst is typically defined as the percentage of released drug from particles in $24 \mathrm{~h}$ immediately after exposure to release media. As expected, the smallest microparticles, fabricated in 5\% (w/v) PVA solution, exhibited an initial burst corresponding to $58.62 \%$ of the encapsulated drug. Unlike microparticles with 5\% (w/v) PVA solution, microparticles with $2 \%(\mathrm{w} / \mathrm{v})$ PVA solution released SKL2020 continuously for 7 days and showed a linear release profile. Microparticles with $0.2 \%$ (w/v) PVA solution showed much slower release rates for the first 4 days and then started to release drug at a higher rate from days 4 to 9 . This difference can be explained by the biphasic nature of the drug release pattern of PLGA particles: diffusion and

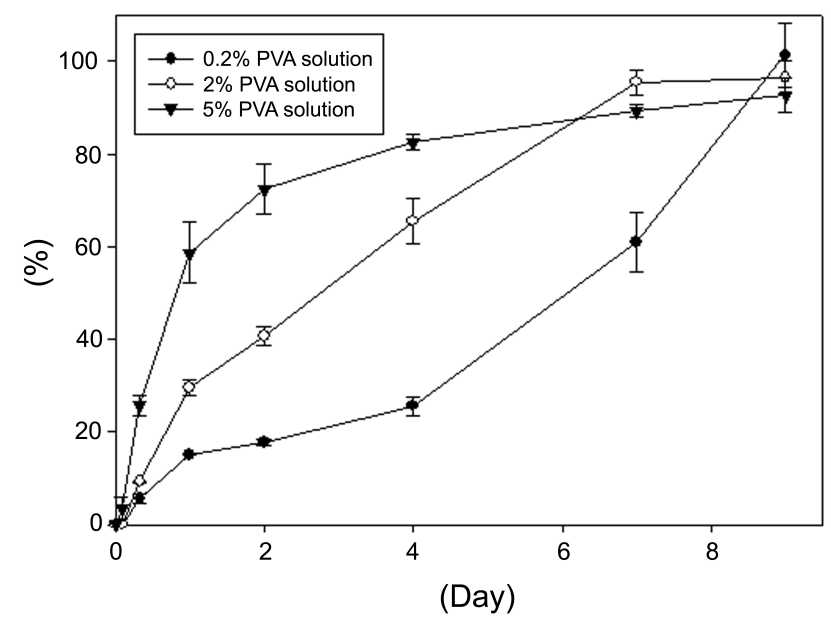

Figure 3. Effect of PVA concentration on SKL-2020 release rates.
Table 2. Characterization of SKL-2020-loaded PLGA microparticles fabricated with different initial drug contents

\begin{tabular}{ccccc}
\hline $\begin{array}{c}\text { Initial drug } \\
\text { content }(\%)\end{array}$ & $\begin{array}{c}\text { Final drug } \\
\text { content }(\%)\end{array}$ & $\begin{array}{c}\text { E.E. } \\
(\%)\end{array}$ & $\begin{array}{c}\text { Mean size } \\
(\mu \mathrm{m})\end{array}$ & $\begin{array}{c}\text { Burst } \\
(\%)\end{array}$ \\
\hline 5 & 1.63 & 34.19 & 10.63 & 61.71 \\
10 & 2.39 & 26.27 & 10.20 & 58.20 \\
20 & 4.28 & 25.67 & 10.89 & 70.05 \\
\hline
\end{tabular}

degradation. When particle size is small and surface area is large, the drug diffusion which is localized along the surface is dominant. Thus, more than $50 \%$ of the encapsulated drug is released by diffusion in $24 \mathrm{~h}$ for small microparticles. However, diffusion is not a major release mechanism for large microparticles and they releases only a small percentage of encapsulated drug by diffusion until polymer matrix begins to degrade. Once polymer degradation is initiated, a large amount of drug is released into the media. On an average, 7-9 days were required for the PLGA microparticles to degrade completely through bulk erosion and to release all encapsulated drugs.

Drug Loading. To study the effect of the ratio of initial drug content to polymer on release properties in $48 \mathrm{~h}$, PLGA microparticles with $5 \%, 10 \%$ and $20 \%$ initial SKL-2020 content were fabricated in 5\%(w/v) PVA solutions. As a result, a decrease in encapsulation efficiency appeared (Table 2). This decreasing trend of encapsulation efficiency with increasing initial drug content can be explained by a larger concentration gradient of SKL-2020 between the dispersed and continuous phase. The concentration gradient is a major driving force for drug diffusion into a water phase during the particle-hardening step. Many previous studies have shown consistency in this result. Although encapsulation efficiency decreased, the final drug content increased almost by twice and 4 times compared with microparticles containing 5\% initial drug content. When the final drug content is high, low amounts of PLGA microparticles are necessary to deliver specific amounts of SKL-2020. This could be very advantageous because it may eliminate side effects caused by using massive amounts of polymer. However, the release profile is the most imprtant in a drug delivery system. Any changes in release patterns with different drug loading methods must be studied.

Although there was no significant difference among cumulative amounts of released drug after $24 \mathrm{~h}$, an initial burst of PLGA microparticles with $20 \%$ initial drug content was observed immediately (Fig. 4). These particles released $56 \%$ of encapsulated drug in only $2 \mathrm{~h}$, nearly 3 times the amount of drug that other microparticles released. If too much drug is released over a short time, the drug delivery system may not be sustainable and potential side effects could arise. However, SKL-2020-loaded PLGA microparticles with $10 \%$ initial drug content showed release patterns similar to those of microparticles with $5 \%$ initial drug content.

Mixtures of Microparticles Prepared with Different Fabrication Conditions. Since there are limits involved in 


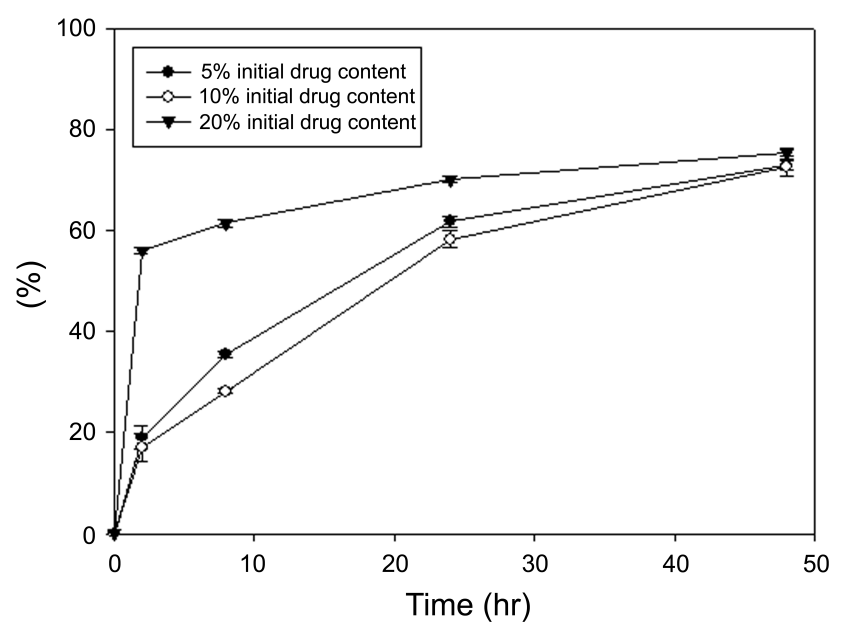

Figure 4. Effect of initial drug content on SKL-2020 release rates.

precise designing of release patterns of uniform microparticles under varying fabrication conditions, mixing microparticles prepared with different fabrication conditions is one option for obtaining desirable release profiles. Generally, a severe initial drug release burst must be avoided to prevent potential risks such as inflammation and poor sustainability of drug release. To reduce the initial burst and maintain the drug release rate for at least 1 week, mixtures of 2 microparticles with unique in vitro release patterns were used. As SKL-2020-loaded PLGA microparticles with $0.2 \%(\mathrm{w} / \mathrm{v})$ PVA solution have no initial burst because of reduced diffusion through the polymer, and SKL-2020-loaded PLGA microparticles with $5 \%(\mathrm{w} / \mathrm{v})$ PVA solution have initial bursts nearly $60 \%$ of total encapsulated drug, a mixture of 2 different microparticles was predicted to provide a new release profile having combined characteristics of the 2 methods. Berkland et al. reported that mixture release profiles correspond to a mass-weighted linear combination of individual release profiles. ${ }^{11}$

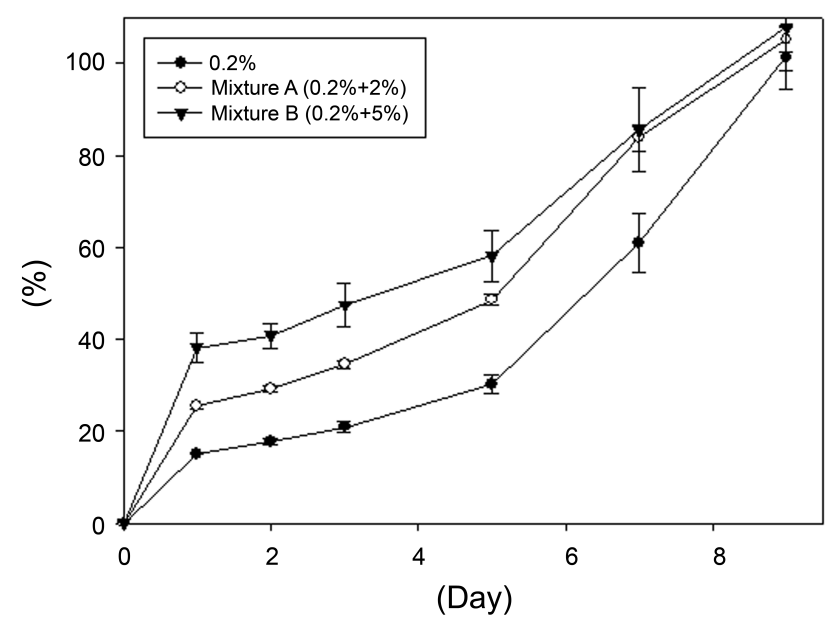

Figure 5. SKL-2020 release from PLGA microparticles fabricated in $0.2 \%$ PVA solution and mixtures of two different microparticles. (Drug weight ratio- Mixture A. 0.2\%:2\%=7:5, Mixture B. 0.2\%: $5 \%=16: 9)$

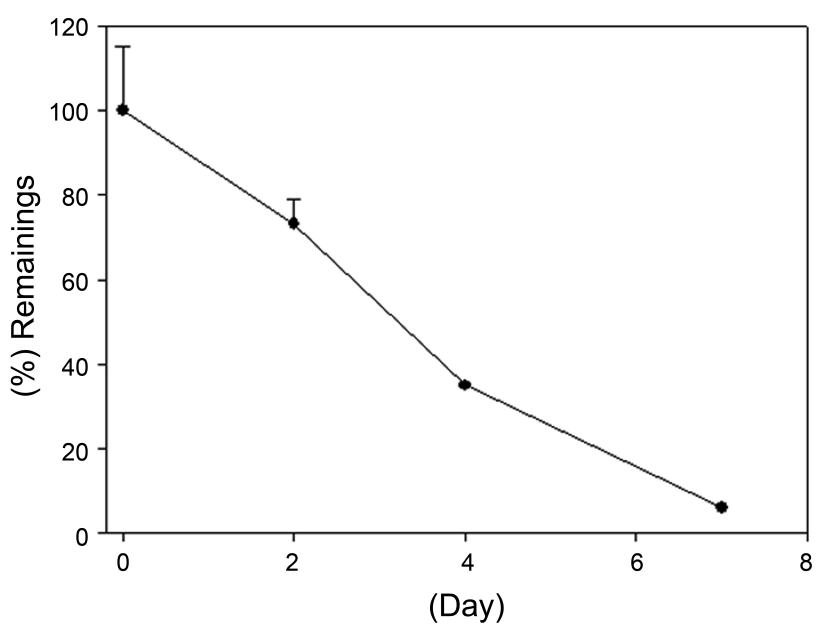

Figure 6. Remained SKL-2020 in PLGA microparticles fabricated in 5\% PVA solution.

When SKL-2020-loaded PLGA microparticles with $0.2 \%$ (w/v) PVA solution were mixed with SKL-2020-loaded PLGA microparticles with $2 \%$ and $5 \%(\mathrm{w} / \mathrm{v})$ PVA solution, the initial burst effect of small particles was efficiently repressed (Fig. 5). Because the amount of drug released by the initial burst was relatively low due to the presence of large particles not contributing to the initial burst, the total initial burst decreased. This method also prevented premature SKL-2020 saturation from microparticles with $2 \%$ and $5 \%(\mathrm{w} / \mathrm{v})$ PVA solution and extended release time with slower release rate. The release profile of the mixture can be altered depending on individual characteristics and weight ratios of differentially prepared microparticles.

In vivo Release Profile. To guarantee the suitability of SKL-2020-loaded PLGA microparticles for the human body, in vivo release was examined. A discrepancy between in vitro and in vivo experimental results has been reported for many cases. The preferable product represents microparticles fabricated with 5\% initial drug loading in 5\% (w/v) PVA solution. It was chosen for in vivo studies because an efficient and immediate drug effect was expected. Additionally, it was necessary to confirm the sustainability of in vivo drug release from microparticles.

The obtained in vivo release profile (Fig. 6) displayed a completely different release pattern compared with the in vitro release profile (Fig. 3). First, a severe initial burst that could cause side effects disappeared; this was followed by a sustained drug release of over 1 week. Since the release pattern was nearly linear, the drug release rate of SKL-2020loaded PLGA microparticles prepared in 5\% (w/v) PVA solution did not change from day 0 to 7 .

To study the efficacy of SKL-2020-loaded PLGA microparticles, the same in vivo experiment with SKL-2020 was performed, too. After 2 days, SKL-2020 was not detected in the adductor muscles of mice (Data not shown). Given that the in vivo half-life of SKL-2020 is short, PLGA microparticles are ideal as a drug delivery system that can guarantee the long-term delivery of SKL-2020. 


\section{Conclusions}

Drug release kinetics can be varied depending on fabrication parameters such as PVA concentration or the amount of drug loading. Microparticle size is a key release pattern determinant affected primarily by PVA concentration. With high PVA concentration, small microparticles were generated and provided undesirable release patterns with an initial burst. Additionally, a large amount of initially loaded drug led to a severe initial burst due to a large concentration gradient, explaining the tendency of decreasing encapsulation efficiency. The in vivo release pattern was inconsistent with the in vitro release pattern. Undesirable burst effects of the in vitro release profile were eliminated in the in vivo release test.

Acknowledgments. This study was supported by a grant of the Korea Healthcare technology R\&D Project, Ministry for Health, Welfare \& Family Affairs, Republic of Korea. (A084285).

\section{References}

1. Choi, H. S.; Seo, S. A.; Khang, G.; Rhee, J. M.; Lee, H. B. Int. J. Pharm. 2002, 234, 195.

2. Xie, S.; Wang, S.; Zhu, L.; Wang, F.; Zhour, W. Colloid. Surface B 2009, 74, 358 .

3. Lee, W. K.; Park, J. Y.; Yang, E. H.; Suh, H.; Kim, S. H.; Chung, D. S.; Choi, K.; Yang, C. W.; Park, J. S. J. Control. Release 2002, $84,115$.

4. Berkland, C.; Kim, K.; Pack, D. W. J. Control. Release 2001, 73, 59.

5. Govender, T.; Stolnik, S.; Garnett, M. C.; Illum, L.; Davis, S. S. J. Control. Release 1999, 57, 171

6. Ye, M.; Kim, S.; Park, K. J. Control. Release 2010, 146, 241.

7. Freitas, S.; Merkle, H. P.; Gander, B. J. Control. Release 2005, $102,313$.

8. Katou, H.; Wandrey, A. J.; Gander, B. Int. J. Pharm. 2008, 364, 45.

9. Yeo, Y.; Park, K. Arch. Pharm. Res. 2004, 27, 1.

10. Yang, Y.; Chung, T.; Ng, N. P. Biomaterials 2001, 22, 231.

11. Berkland, C.; King, M.; Cox, A.; Kim, K.; Pack, D. W. J. Control. Release 2002, 82, 137. 